\title{
Does the Equivalence between Gravitational Mass and Energy Survive for a Composite Quantum Body?
}

\author{
A. G. Lebed ${ }^{1,2}$ \\ ${ }^{1}$ Department of Physics, University of Arizona, 1118 E. 4th Street, Tucson, AZ 85721, USA \\ ${ }^{2}$ L. D. Landau Institute for Theoretical Physics, 2 Kosygina Street, Moscow 117334, Russia
}

Correspondence should be addressed to A. G. Lebed; lebed@physics.arizona.edu

Received 7 November 2013; Accepted 21 January 2014; Published 9 March 2014

Academic Editor: Douglas Singleton

Copyright (C) 2014 A. G. Lebed. This is an open access article distributed under the Creative Commons Attribution License, which permits unrestricted use, distribution, and reproduction in any medium, provided the original work is properly cited. The publication of this article was funded by SCOAP ${ }^{3}$.

We define passive and active gravitational mass operators of the simplest composite quantum body-a hydrogen atom. Although they do not commute with its energy operator, the equivalence between the expectation values of passive and active gravitational masses and energy is shown to survive for stationary quantum states. In our calculations of passive gravitational mass operator, we take into account not only kinetic and Coulomb potential energies but also the so-called relativistic corrections to electron motion in a hydrogen atom. Inequivalence between passive and active gravitational masses and energy at a macroscopic level is demonstrated to reveal itself as time-dependent oscillations of the expectation values of the gravitational masses for superpositions of stationary quantum states. Breakdown of the equivalence between passive gravitational mass and energy at a microscopic level reveals itself as unusual electromagnetic radiation, emitted by macroscopic ensemble of hydrogen atoms, moved by small spacecraft with constant velocity in the Earth's gravitational field. We suggest the corresponding experiment on the Earth's orbit to detect this radiation, which would be the first direct experiment where quantum effects in general relativity are observed.

\section{Introduction}

Formulation of a successful quantum gravitational theory is considered to be one of the most important problems in physics and the major step towards the so-called "Theory of Everything." On the other hand, fundamentals of general relativity and quantum mechanics are so different that it is possible that these two theories will not be united in the feasible future. In this difficult situation, it seems to be important to suggest a combination of quantum mechanics and some nontrivial approximation of general relativity. In particular, this is important in the case where such theory leads to meaningful physical results, which can be experimentally tested.

A notion of gravitational mass of a composite body is known to be nontrivial in general relativity and related to the following paradoxes. If we consider a free photon with energy $E$ and apply to it the so-called Tolman's formula for active gravitational mass (see, e.g., [1]), we will obtain $m_{a}^{g}=$ $2 E / c^{2}$ (i.e., two times bigger value than the expected one) [2].
If a photon is confined in a box with mirrors, then we have a composite body at rest. In this case, as shown in [2], we have to take into account a negative contribution to $m_{a}^{g}$ from stress in the box walls to restore Einstein's equation, $m_{a}^{g}=E / c^{2}$. It is important that the latter equation is restored only after averaging over time. A role of the classical virial theorem in establishing the equivalence between averaged over time active and passive gravitational masses and energy is discussed in detail in $[3,4]$ for different types of classical composite bodies. In particular, for electrostatically bound two bodies, it is shown that gravitational field is coupled to a combination $3 K+2 U$, where $K$ is kinetic and $U$ is the Coulomb potential energies. Since the classical virial theorem states that the following time average is equal to zero, $\langle 2 K+U\rangle_{t}=0$, then we conclude that averaged over time active and passive gravitational masses are proportional to the total amount of energy $[3,4]$,

$$
\left\langle m_{a, p}^{g}\right\rangle_{t}=m_{1}+m_{2}+\frac{\langle 3 K+2 U\rangle_{t}}{c^{2}}=\frac{E}{c^{2}},
$$


where $m_{1}$ and $m_{2}$ are bare masses of the above considered bodies.

\section{Goal}

The main goal of our paper is to study a quantum problem about passive [5-7] and active gravitational masses of a composite body. As the simplest example, we consider a hydrogen atom. We claim four main results in the paper. Our first result is that the equivalence between passive and active gravitational masses and energy survives at a macroscopic level for stationary quantum states. In the calculations of passive gravitational mass operator, we take into account both nonrelativistic kinetic and Coulomb potential energies and the so-called relativistic corrections (see, e.g., [8]) to an electron motion in a hydrogen atom, whereas in calculations of active gravitational mass we take into account only nonrelativistic kinetic and Coulomb potential energies. More specifically, we show that the expectation values of passive and active gravitational masses of the atom are equivalent to its energy for stationary quantum states due to some mathematical theorems. In the case of active gravitational mass, the corresponding theorem is known as the quantum virial theorem (see, e.g., [9]), whereas, in the case of passive gravitational mass, the corresponding theorem is more complicated than that in [9]. In fact the latter is an extension of the relativistic quantum virial theorem [10] for the case of a particle with spin $1 / 2$. We would like to draw attention to the fact that the abovementioned results are nontrivial. Indeed, below we define passive and active gravitational mass operators of an electron, $\widehat{m}_{p}^{g}$ and $\widehat{m}_{a}^{g}$, respectively, in the post-Newtonian approximation to general relativity. It is important that these operators occur not to commute with electron energy operator, taken in the absence of the field. Therefore, from the first point of view, it seems that the equivalence between passive and active gravitational masses and energy is broken. Nevertheless, using rather sophisticated mathematical tools, we show that the expectation values of passive and active gravitational mass operators are $\left\langle\widehat{m}_{p}^{g}\right\rangle=$ $\left\langle\widehat{m}_{a}^{g}\right\rangle=m_{e}+E_{n} / c^{2}$ for stationary quantum states in a hydrogen atom, where $m_{e}$ is the bare electron mass and $E_{n}$ is the total electron energy of $n$th atomic energy level.

Our second result is that the equivalence between electron energy and its passive and active gravitational masses is shown to be broken for superpositions of stationary quantum states. More strictly speaking, we demonstrate that there exist such quantum states where the expectation values of energy are constant, whereas the expectation values of passive and active gravitational masses are oscillatory functions of time. Our third result is a breakdown of the equivalence between passive gravitational mass and energy at a microscopic level. It is a consequence of the fact that passive electron gravitational mass operator, $\widehat{m}_{p}^{g}$, does not commute with its energy operator, taken in the absence of the field. Therefore, an atom with a definite energy in the absence of gravitational field, $E$, is not characterized by a definite passive gravitational mass in an external gravitational field. Passive gravitational mass is shown to be quantized and can significantly differ from the value $E / c^{2}$. Our fourth result is that we suggest how the abovementioned inequivalence can be experimentally observed. In particular, we propose experimental detection of electromagnetic radiation, emitted by macroscopic ensemble of hydrogen atoms (in a real experiment-molecules), supported by and moving with constant velocity in the Earth's gravitational field, using small spacecraft or satellite. If such experiment is done, to the best of our knowledge, it will be the first direct experimental test of quantum effects in general relativity. We stress that so far only quantum effects in the Newtonian variant of gravity, where general relativity corrections are negligible, have been directly studied in the famous COW [11] and ILL [12] experiments.

Most of the abovementioned results, related to passive gravitational mass, have been recently published by us in [57]. All results, related to active gravitational mass, and results, related to the so-called relativistic corrections to passive gravitational mass, are new and, to the best of our knowledge, have not been published.

\section{Equivalence of the Expectation Values of Passive Gravitational Mass and Energy for Stationary States}

Let us use the standard weak field approximation to describe spacetime outside the Earth (see, e.g., [13]). (We pay attention to the fact that, to calculate the Hamiltonian in a linear with respect to a small parameter $\phi(R) / c^{2}$ approximation, we do not need to keep the terms of the order of $\left[\phi(R) / c^{2}\right]^{2}$ in the metric (2), in contrast to the classical problem about perihelion precession of Mercury's orbit [13].),

$$
\begin{array}{r}
d s^{2}=-\left(1+\frac{2 \phi}{c^{2}}\right)(c d t)^{2}+\left(1-\frac{2 \phi}{c^{2}}\right)\left(d x^{2}+d y^{2}+d z^{2}\right), \\
\phi=-\frac{G M}{R},
\end{array}
$$

where $G$ is the gravitational constant, $c$ is the velocity of light, $M$ is the Earth's mass, and $R$ is a distance from center of the Earth. Then, in the local proper spacetime coordinates:

$$
\begin{aligned}
x^{\prime} & =\left(1-\frac{\phi}{c^{2}}\right) x, & y^{\prime} & =\left(1-\frac{\phi}{c^{2}}\right) y, \\
z^{\prime} & =\left(1-\frac{\phi}{c^{2}}\right) z, & t^{\prime} & =\left(1+\frac{\phi}{c^{2}}\right) t,
\end{aligned}
$$

the Schrödinger equation for an electron motion in a hydrogen atom can be approximately written in the following standard form:

$$
i \hbar \frac{\partial \Psi\left(\mathbf{r}^{\prime}, t^{\prime}\right)}{\partial t^{\prime}}=\widehat{H}\left(\widehat{\mathbf{p}^{\prime}}, \mathbf{r}^{\prime}\right) \Psi\left(\mathbf{r}^{\prime}, t^{\prime}\right),
$$

where $\widehat{H}\left(\widehat{\mathbf{p}^{\prime}}, \mathbf{r}^{\prime}\right)$ is the standard Hamiltonian. We stress that, in (4) and everywhere below, we disregard all tidal effects (i.e., we do not differentiate gravitational potential with respect 
to electron coordinates, $\mathbf{r}$ and $\mathbf{r}^{\prime}$, corresponding to electron positions in the center of mass coordinate systems). It is possible to show that this means that we consider a hydrogen atom as a point-like body and disregard all tidal terms in electron Hamiltonian, which are usually very small and of the order of $\left(r_{B} / R_{0}\right)\left|\phi / c^{2}\right| \sim 10^{-17}\left|\phi / c^{2}\right| \sim 10^{-26}$ in the Earth's gravitational field. (Here $r_{B}$ is the so-called Bohr's radius and $R_{0}$ is the Earth's radius.)

3.1. Nonrelativistic Case. Let us first consider nonrelativistic Schrödinger equation for electron motion in a hydrogen atom, where we take into account only kinetic and Coulomb potential energies:

$$
\begin{gathered}
i \hbar \frac{\partial \Psi\left(\mathbf{r}^{\prime}, t^{\prime}\right)}{\partial t^{\prime}}=\widehat{H}_{0}\left(\widehat{\mathbf{p}^{\prime}}, \mathbf{r}^{\prime}\right) \Psi\left(\mathbf{r}^{\prime}, t^{\prime}\right), \\
\widehat{H}_{0}\left(\widehat{\mathbf{p}^{\prime}}, \mathbf{r}^{\prime}\right)=m_{e} c^{2}+\frac{\widehat{\mathbf{p}}^{2}}{2 m_{e}}-\frac{e^{2}}{r^{\prime}},
\end{gathered}
$$

where $e$ is the electron charge, $r^{\prime}$ is a distance between electron and proton, and $\widehat{\mathbf{p}^{\prime}}=-i \hbar \partial / \partial \mathbf{r}^{\prime}$ is electron momentum operator in the local proper spacetime coordinates. Below, we treat the weak gravitational field (2) as a perturbation in inertial coordinate system, corresponding to spacetime coordinates $(x, y, z, t)$ in $(3)[3,4]$, and calculate the corresponding Hamiltonian:

$$
\begin{aligned}
\widehat{H}_{0}(\widehat{\mathbf{p}}, \mathbf{r}, \phi)= & m_{e} c^{2}+\frac{\widehat{\mathbf{p}}^{2}}{2 m_{e}}-\frac{e^{2}}{r} \\
& +m_{e} \phi+\left(3 \frac{\widehat{\mathbf{p}}^{2}}{2 m_{e}}-2 \frac{e^{2}}{r}\right) \frac{\phi}{c^{2}} .
\end{aligned}
$$

From (6), it is clear that the Hamiltonian can be rewritten in the following form:

$$
\widehat{H}_{0}(\widehat{\mathbf{p}}, \mathbf{r}, \phi)=m_{e} c^{2}+\frac{\widehat{\mathbf{p}}^{2}}{2 m_{e}}-\frac{e^{2}}{r}+\widehat{m}_{e}^{g} \phi,
$$

where we introduce passive gravitational mass operator of an electron:

$$
\begin{aligned}
\widehat{m}_{p}^{g}= & m_{e}+\frac{\left(\left(\widehat{\mathbf{p}}^{2} / 2 m_{e}\right)-\left(e^{2} / r\right)\right)}{c^{2}} \\
& +\frac{\left(\left(2\left(\hat{\mathbf{p}}^{2} / 2 m_{e}\right)\right)-\left(e^{2} / r\right)\right)}{c^{2}},
\end{aligned}
$$

which is proportional to its weight operator in the weak gravitational field (2). (Note that passive gravitational mass of a composite body, as a measurable quantity, has to be represented in quantum mechanics by some Hermitian operator. The most logical and straightforward way is to define the passive gravitational mass operator to be proportional to a weight operator in a weak gravitational field (i.e., the operator which couples with gravitational potential in a linear approximation in (6)). This extends definitions of gravitational mass in classical case $[3,4]$ and corresponds to the so-called Eötvös method to measure gravitational mass [13].). Note that, in (8), the first term corresponds to the bare electron mass, $m_{e}$, and the second term corresponds to the expected electron energy contribution to the gravitational mass operator, whereas the third nontrivial term is the virial contribution to passive gravitational mass operator. It is possible to make sure $[5,14]$ that $(7)$ and $(8)$ can be obtained directly from the Dirac equation in a curved spacetime, corresponding to the weak centrosymmetric gravitational field (2) (see, e.g., (3.24) in [15]), if we disregard all tidal terms).

Here, we discuss some important consequence of (7) and (8). It is crucial that the operator (8) does not commute with electron energy operator, taken in the absence of gravitational field. Therefore, it is not clear from the beginning that the equivalence between electron passive gravitational mass and its energy exists. To establish the equivalence at a macroscopic level, we consider a macroscopic ensemble of hydrogen atoms with each of them being in a stationary quantum state with a definite energy $E_{n}$. Then, from (8), it follows that the expectation value of electron passive gravitational mass operator per atom is

$$
\begin{aligned}
\left\langle\widehat{m}_{p}^{g}\right\rangle & =m_{e}+\frac{E_{n}}{c^{2}}+\frac{\left\langle\left(2\left(\widehat{\mathbf{p}}^{2} / 2 m_{e}\right)\right)-\left(e^{2} / r\right)\right\rangle}{c^{2}} \\
& =m_{e}+\frac{E_{n}}{c^{2}},
\end{aligned}
$$

where the third term in (9) is zero in accordance with the quantum virial theorem [9]. Therefore, we conclude that the equivalence between passive gravitational mass and energy survives at a macroscopic level for stationary quantum states, if we consider only pairings of nonrelativistic kinetic and Coulomb potential energies with an external gravitational field. Note that an important difference between our result (9) and the corresponding result in classical case $[3,4]$ is that the expectation value of passive gravitational mass corresponds to averaging procedure over a macroscopic ensemble of hydrogen atoms, whereas in classical case we average over time.

3.2. Relativistic Corrections. In this section, we study a more general case, where the so-called relativistic corrections to an electron motion in a hydrogen atom are taken into account. As well-known [8], there exist three relativistic correction terms, which have different physical meanings. The total Hamiltonian in the absence of gravitational field can be written as

$$
\widehat{H}(\widehat{\mathbf{p}}, \mathbf{r})=\widehat{H}_{0}(\widehat{\mathbf{p}}, \mathbf{r})+\widehat{H}_{1}(\widehat{\mathbf{p}}, \mathbf{r}),
$$

where

$$
\widehat{H}_{1}(\widehat{\mathbf{p}}, \mathbf{r})=\alpha \widehat{\mathbf{p}}^{4}+\beta \delta^{3}(\mathbf{r})+\gamma \frac{\widehat{\mathbf{s}} \cdot \widehat{\mathbf{L}}}{r^{3}},
$$

with the parameters $\alpha, \beta$, and $\gamma$ being

$$
\alpha=-\frac{1}{8 m_{e}^{3} c^{2}}, \quad \beta=\frac{\pi e^{2} \hbar^{2}}{2 m_{e}^{2} c^{2}}, \quad \gamma=\frac{e^{2}}{2 m_{e}^{2} c^{2}} .
$$


(Here, $\delta^{3}(\mathbf{r})=\delta(x) \delta(y) \delta(z)$ is a three-dimensional Dirac's delta function.) Note that the first contribution in (11) is called the kinetic term, the second one is the so-called Darwin's term, and the third one is the spin-orbital interaction, where $\widehat{\mathbf{L}}=-i \hbar[\mathbf{r} \times \partial / \partial \mathbf{r}]$ is electron angular momentum operator. In the presence of the weak gravitational field (2), the Schrödinger equation for an electron motion in the local proper spacetime coordinates (3) can be approximately written as

$$
i \hbar \frac{\partial \Psi\left(\mathbf{r}^{\prime}, t^{\prime}\right)}{\partial t^{\prime}}=\left[\widehat{H}_{0}\left(\widehat{\mathbf{p}^{\prime}}, \mathbf{r}^{\prime}\right)+\widehat{H}_{1}\left(\widehat{\mathbf{p}^{\prime}}, \mathbf{r}^{\prime}\right)\right] \Psi\left(r^{\prime}, t^{\prime}\right) .
$$

(Note that, as discussed above, we disregard everywhere all tidal effects.)

By means of the coordinates transformation (3), the corresponding Hamiltonian in inertial coordinate system $(x, y, z, t)$ can be expressed as

$$
\begin{aligned}
\widehat{H}(\widehat{\mathbf{p}}, \mathbf{r}, \phi) & \\
= & {\left[\widehat{H}_{0}(\widehat{\mathbf{p}}, \mathbf{r})+\widehat{H}_{1}(\widehat{\mathbf{p}}, \mathbf{r})\right]\left(1+\frac{\phi}{c^{2}}\right) } \\
& +\left(2 \frac{\widehat{\mathbf{p}}^{2}}{2 m_{e}}-\frac{e^{2}}{r}+4 \alpha \widehat{\mathbf{p}}^{4}+3 \beta \delta^{3}(\mathbf{r})+3 \gamma \frac{\widehat{\mathbf{S}} \cdot \widehat{\mathbf{L}}}{r^{3}}\right) \frac{\phi}{c^{2}} .
\end{aligned}
$$

For the Hamiltonian (14), passive gravitational mass operator of an electron can be written in a more complicated form than (8):

$$
\begin{aligned}
& \widehat{m}_{p}^{g} \\
& =m_{e} \\
& +\frac{\left(\left(\widehat{\mathbf{p}}^{2} / 2 m_{e}\right)-\left(e^{2} / r\right)+\alpha \widehat{\mathbf{p}}^{4}+\beta \delta^{3}(\mathbf{r})+\left(\gamma\left((\widehat{\mathbf{S}} \cdot \widehat{\mathbf{L}}) / r^{3}\right)\right)\right)}{c^{2}} \\
& +\frac{\left(\left(2\left(\widehat{\mathbf{p}}^{2} / 2 m_{e}\right)\right)-\left(e^{2} / r\right)+4 \alpha \widehat{\mathbf{p}}^{4}+3 \beta \delta^{3}(\mathbf{r})+\left(3 \gamma\left((\widehat{\mathbf{S}} \cdot \widehat{\mathbf{L}}) / r^{3}\right)\right)\right)}{c^{2}} .
\end{aligned}
$$

Let us consider again a macroscopic ensemble of hydrogen atoms with each of them being in a stationary quantum state with a definite energy $E_{n}^{\prime}$, where $E_{n}^{\prime}$ takes into account the relativistic corrections (11) to electron energy. In this case, the expectation value of the electron mass operator (15) per atom can be written as

$$
\begin{aligned}
& \left\langle\widehat{m}_{p}^{g}\right\rangle \\
& =m_{e}+\frac{E_{n}^{\prime}}{c^{2}} \\
& +\frac{\left\langle\left(2\left(\widehat{\mathbf{p}}^{2} / 2 m_{e}\right)\right)-\left(e^{2} / r\right)+4 \alpha \widehat{\mathbf{p}}^{4}+3 \beta \delta^{3}(\mathbf{r})+\left(3 \gamma\left((\widehat{\mathbf{S}} \cdot \widehat{\mathbf{L}}) / r^{3}\right)\right)\right\rangle}{c^{2}} .
\end{aligned}
$$

Below, we show that the expectation value of the third term in (16) is zero and, therefore, Einstein's equation, related to the expectation value of passive gravitational mass and energy, can be applied to stationary quantum states. Here, we define the so-called virial operator [9] as

$$
\widehat{G}=\frac{1}{2}(\widehat{\mathbf{p}} \mathbf{r}+\mathbf{r} \widehat{\mathbf{p}}),
$$

and write the standard equation of motion for its expectation value:

$$
\frac{d}{d t}\langle\widehat{G}\rangle=\frac{i}{\hbar}\left\langle\left[\widehat{H}_{0}(\widehat{\mathbf{p}}, \mathbf{r})+H_{1}(\widehat{\mathbf{p}}, \mathbf{r}), \widehat{G}\right]\right\rangle,
$$

where $[\widehat{A}, \widehat{B}]$, as usual, stands for a commutator of two operators, $\widehat{A}$ and $\widehat{B}$. If we consider a stationary quantum state with a definite energy, $E_{n}^{\prime}$, then the derivative $d\langle\widehat{G}\rangle / d t$ in (18) has to be zero and, thus,

$$
\left\langle\left[\widehat{H}_{0}(\widehat{\mathbf{p}}, \mathbf{r})+H_{1}(\widehat{\mathbf{p}}, \mathbf{r}), \widehat{G}\right]\right\rangle=0,
$$

where the Hamiltonians $\widehat{H}_{0}(\widehat{\mathbf{p}}, \mathbf{r})$ and $\widehat{H}_{1}(\widehat{\mathbf{p}}, \mathbf{r})$ are defined by (5) and (11). By means of rather lengthy but straightforward calculations it is possible to show that

$$
\begin{gathered}
\frac{\left[\widehat{H}_{0}(\widehat{\mathbf{p}}, \mathbf{r}), \widehat{G}\right]}{-i \hbar}=2 \frac{\widehat{\mathbf{p}}^{2}}{2 m_{e}}-\frac{e^{2}}{r}, \quad \frac{\left[\alpha \widehat{\mathbf{p}}^{4}, \widehat{G}\right]}{-i \hbar}=4 \alpha \widehat{\mathbf{p}}^{4}, \\
\frac{\left[\beta \delta^{3}(\mathbf{r}), \widehat{G}\right]}{-i \hbar}=3 \beta \delta^{3}(\mathbf{r}), \quad \frac{1}{-i \hbar}\left[\gamma \frac{\widehat{\mathbf{S}} \cdot \widehat{\mathbf{L}}}{r^{3}}, \widehat{G}\right]=3 \gamma \frac{\widehat{\mathbf{S}} \cdot \widehat{\mathbf{L}}}{r^{3}},
\end{gathered}
$$

where we take into account the following equality: $x_{i}\left(d \delta\left(x_{i}\right) /\right.$ $\left.d x_{i}\right)=-\delta\left(x_{i}\right)$. As directly follows from (19) and (20):

$$
\left\langle 2 \frac{\widehat{\mathbf{p}}^{2}}{2 m_{e}}-\frac{e^{2}}{r}+4 \alpha \widehat{\mathbf{p}}^{4}+3 \beta \delta^{3}(\mathbf{r})+3 \gamma \frac{\widehat{\mathbf{S}} \cdot \widehat{\mathbf{L}}}{r^{3}}\right\rangle=0,
$$

and, therefore, (16) can be rewritten in Einstein's form:

$$
\left\langle\widehat{m}_{p}^{g}\right\rangle=m_{e}+\frac{E_{n}^{\prime}}{c^{2}}
$$

(Note that (21) extends the so-called relativistic quantum virial theorem [10], derived for spinless particles, to the case of particles with spin $1 / 2$.)

It is important that (22) directly establishes the equivalence between the expectation value of electron passive gravitational mass and its energy in a hydrogen atom, including the relativistic corrections, for the Eötvös' type of experiments [13]. We speculate that such equivalence exists also for more complicated quantum systems, including manybody systems with arbitrary interactions of particles. These reveal and establish the physical meaning of a coupling of a macroscopic quantum test body with a weak gravitational field.

\section{Inequivalence between Passive Gravitational Mass and Energy for Superpositions of Stationary States}

In the previous section, we have shown that the expectations values of passive gravitational mass and energy are equivalent to each other in stationary quantum states. Here, we 
investigate if such equivalence survives for superpositions of stationary quantum states. For this purpose, we consider the simplest superposition of the ground and first excited $s$-wave states in a hydrogen atom, where electron wave function has the following form:

$$
\Psi_{1,2}(r, t)=\frac{1}{\sqrt{2}}\left[\Psi_{1}(r) \exp \left(-i E_{1} t\right)+\Psi_{2}(r) \exp \left(-i E_{2} t\right)\right]
$$

It is important that wave function (23) is characterized by the time-independent expectation value of energy, $\langle E\rangle=$ $\left(E_{1}+E_{2}\right) / 2$. Nevertheless, the expectation value of passive gravitational mass operator (8) occurs to be the following time-dependent oscillatory function:

$$
\left\langle\widehat{m}_{p}^{g}\right\rangle=m_{e}+\frac{E_{1}+E_{2}}{2 c^{2}}+\frac{V_{1,2}}{c^{2}} \cos \left[\frac{\left(E_{1}-E_{2}\right) t}{\hbar}\right],
$$

where $V_{1,2}$ is a matrix element of the virial operator:

$$
V_{1,2}=\int \Psi_{1}^{*}(r)\left(2 \frac{\widehat{\mathbf{p}}^{2}}{2 m_{e}}-\frac{e^{2}}{r}\right) \Psi_{2}(r) d^{3} \mathbf{r} .
$$

Note that the above obtained result holds both for nonrelativistic passive gravitational mass operator (8) and for the operator (15), which takes into account the so-called relativistic corrections (11). Therefore, we make a conclusion that the oscillations of passive gravitation mass (24) directly demonstrate inequivalence of passive gravitational mass and energy at a macroscopic level at any given moment of time.

Let us discuss a relative magnitude of the oscillations (24). By using actual numbers for a hydrogen atom, it is possible to obtain the following numerical value of the matrix element of the virial operator: $V_{1,2}=5.7 \mathrm{eV}$. Since $m_{e} c^{2} \simeq 0.5 \mathrm{MeV}$ and $m_{p} \simeq 1800 m_{e}$, we can come to the conclusion that the oscillations (24) are weak but not negligible: $\delta m_{e} / m_{e} \sim$ $10^{-5}$ and $\delta m_{e} / m_{p} \sim 10^{-8}$. They correspond to the following angular and linear frequencies: $\omega_{1,2} \simeq 1.6 \times 10^{16} \mathrm{~Hz}$ and $\nu \simeq$ $2.5 \times 10^{15} \mathrm{~Hz}$, respectively. We hope that the abovementioned oscillations of passive gravitational mass are experimentally measured, despite the fact that the quantum state (23) decays with time.

On the other hand, if we average the oscillations (24) over time, we obtain the modified equivalence principle between the averaged over time expectation value of passive gravitational mass and the expectation value of energy in the following form:

$$
\left\langle\left\langle\widehat{m}_{p}^{g}\right\rangle\right\rangle_{t}=m_{e}+\frac{\left(E_{1}+E_{2}\right)}{2 c^{2}}=\left\langle\frac{E}{c^{2}}\right\rangle .
$$

We pay attention that the physical meaning of averaging procedure in (26) is completely different from that for classical time averaging procedure (1) and does not have the corresponding classical analogs.

\section{Breakdown of the Equivalence between Passive Gravitational Mass and Energy at a Microscopic Level}

In this section, we study how noncommutation of passive gravitational mass operators (8) and (15) and the corresponding energy operators, taken in the absence of gravitational field, results in a breakdown of the equivalence between passive gravitational mass and energy. This conclusion does not depend on the relativistic corrections (11); therefore, for certainty, below we consider passive gravitational mass operator in the form of (8). The physical meaning of the abovementioned breakdown is that an electron in its ground state with a definite energy, $m_{e} c^{2}+E_{1}$, is not characterized by a definite passive gravitational mass and, thus, measurements of the mass can give values, which are not related to electron energy by Einstein's equation, $m_{p}^{g} \neq m_{e}+E_{1} / c^{2}$. As we show below, the passive electron gravitational mass values in a hydrogen atom are quantized: $m_{p}^{g}=m_{e}+E_{n} / c^{2}$, where $E_{n}$ is energy corresponding to $n$th energy level.

5.1. First Thought Experiment. Here, we describe the first thought experiment, illustrating inequivalence of energy and passive gravitational mass at a microscopic level. Suppose that we create quantum state of a hydrogen atom with a definite energy in the absence of a gravitational field and then adiabatically switch on the gravitational field (2). More specifically, at $t \rightarrow-\infty$ (i.e., in the absence of gravitational field), a hydrogen atom is in its ground state with wave function,

$$
\Psi_{1}(r, t)=\Psi_{1}(r) \exp \left(-\frac{i m_{e} c^{2} t}{\hbar}-\frac{i E_{1} t}{\hbar}\right)
$$

whereas, in the vicinity of $t=0$ (i.e., in the presence of the gravitational field (2)), it is characterized by the following wave function:

$$
\Psi(r, t)=\sum_{n=1}^{\infty} a_{n}(t) \Psi_{n}(r) \exp \left(-\frac{i m_{e} c^{2} t}{\hbar}-\frac{i E_{n} t}{\hbar}\right) .
$$

(Here, $\Psi_{n}(r)$ is a normalized electron wave function in a hydrogen atom in the absence of gravitational field, corresponding to energy $E_{n}$ (due to symmetry of our problem, we need to keep in (28) only wave functions, corresponding to $n S$ quantum states).)

As follows from (7) and (8), adiabatically switched on gravitational field corresponds to the following timedependent small perturbation:

$$
\begin{aligned}
\widehat{U}(\mathbf{r}, R, t) & \\
=\phi(R)\left[m_{e}+\frac{\left(\left(\widehat{\mathbf{p}}^{2} / 2 m_{e}\right)-\left(e^{2} / r\right)\right)}{c^{2}}\right. & \\
& \left.+\frac{\left(\left(2\left(\widehat{\mathbf{p}}^{2} / 2 m_{e}\right)\right)-\left(e^{2} / r\right)\right)}{c^{2}}\right] \exp (\lambda t),
\end{aligned}
$$


where $\lambda \rightarrow 0$. (Note that our choice of adiabatically switched on gravitational potential (29) with $\lambda \rightarrow 0$ allows to avoid extra velocity-dependent terms (see, e.g., the Largangian in [3]).) The standard calculations by means of the timedependent quantum mechanical perturbation theory [8] give the following results:

$$
\begin{gathered}
a_{1}(t)=\exp \left[-\frac{i \phi(R) m_{e} c^{2} t+i \phi(R) E_{1} t}{c^{2} \hbar}\right], \\
a_{n}(0)=-\frac{\phi(R)}{c^{2}} \frac{V_{n, 1}}{E_{n}-E_{1}}, \quad n \neq 1,
\end{gathered}
$$

where

$$
V_{n, 1}=\int \Psi_{n}^{*}(r)\left(2 \frac{\widehat{\mathbf{p}}^{2}}{2 m_{e}}-\frac{e^{2}}{r}\right) \Psi_{1}(r) d^{3} \mathbf{r}
$$

(Note that the perturbation (29) is characterized by the following selection rule. Electron from $1 S$ ground state of a hydrogen atom can be excited only into $n S$ excited state.)

Let us discuss (30)-(32). It is important that (30) corresponds to the well-known red shift of atomic ground state energy $E_{1}$ in the gravitational field (2). On the other hand, (31) demonstrates that there is a finite probability,

$$
P_{n}=\left|a_{n}(0)\right|^{2}=\left[\frac{\phi(R)}{c^{2}}\right]^{2}\left(\frac{V_{n, 1}}{E_{n}-E_{1}}\right)^{2}, \quad n \neq 1,
$$

that, at $t=0$, electron occupies $n$th energy level. In fact, this means that measurements of gravitational mass (8) in a quantum state with definite energy (27) give the following quantized values:

$$
m_{p}^{g}(n)=m_{e}+\frac{E_{n}}{c^{2}}
$$

with the probabilities (33) for $n \neq 1$. Note that although the probabilities (33) are quadratic with respect to gravitational potential and, thus, small, the corresponding changes of gravitational mass (34) are large and of the order of $\alpha^{2} m_{e}$, where $\alpha$ is the fine structure constant. It is important that the excited levels of a hydrogen atom spontaneously decay; therefore, one can detect the above discussed quantization law of gravitational mass (34) by measuring electromagnetic radiation, emitted by a macroscopic ensemble of hydrogen atoms.

5.2. Second Thought Experiment. Let us discuss the second thought experiment, which directly demonstrates inequivalence between energy and passive gravitational mass at a microscopic level. Suppose that, at $t=0$, we create a ground state wave function of a hydrogen atom, corresponding to the absence of gravitational field (see (27)). Then, in the presence of the gravitational field (2), the wave function (27) is not anymore a ground state of the Hamiltonian (7) and (8), where we treat gravitational field as a small perturbation in inertial coordinate system $[3,4,15]$. It is important that, for an inertial observer, in accordance with (3), a general solution of the Schrödinger equation, corresponding to the Hamiltonian (7) and (8), can be written as

$$
\begin{aligned}
\Psi(r, t)= & \left(1-\frac{\phi}{c^{2}}\right)^{3 / 2} \sum_{n=1}^{\infty} a_{n} \Psi_{n}\left[\left(1-\frac{\phi}{c^{2}}\right) r\right] \\
& \times \exp \left[\frac{-i m_{e} c^{2}\left(1+\phi / c^{2}\right) t}{\hbar}\right] \\
& \times \exp \left[\frac{-i E_{n}\left(1+\phi / c^{2}\right) t}{\hbar}\right] .
\end{aligned}
$$

We pay attention to the fact that wave function (35) is a series of eigenfunctions of passive gravitational mass operator (8), if we take into account only linear terms with respect to small parameter $\phi / c^{2}$. (Here, factor $1-\phi / c^{2}$ is due to a curvature of space, whereas the term $E_{n}\left(1+\phi / c^{2}\right)$ represents the famous red shift in gravitational field. We also pay attention to the fact that the wave function (35) contains a normalization factor $\left(1-\phi / c^{2}\right)^{3 / 2}$.)

In accordance with the basic principles of the quantum mechanics, probability that, at $t>0$, an electron occupies excited state with energy $m_{e} c^{2}\left(1+\phi / c^{2}\right)+E_{n}\left(1+\phi / c^{2}\right)$ is

$$
\begin{gathered}
P_{n}=\left|a_{n}\right|^{2}, \\
a_{n}=\int \Psi_{1}^{*}(r) \Psi_{n}\left[\left(1-\frac{\phi}{c^{2}}\right) r\right] d^{3} \mathbf{r} \\
=-\left(\frac{\phi}{c^{2}}\right) \int \Psi_{1}^{*}(r) r \Psi_{n}^{\prime}(r) d^{3} \mathbf{r} .
\end{gathered}
$$

(Note that it is possible to demonstrate that for $a_{1}$ in (36) a linear term with respect to gravitational potential, $\phi$, is zero, which is a consequence of the quantum virial theorem.) Taking into account that the Hamiltonian is a Hermitian operator, it is possible to show that, for $n \neq 1$,

$$
\int \Psi_{1}^{*}(r) r \Psi_{n}^{\prime}(r) d^{3} \mathbf{r}=\frac{V_{n, 1}}{\left(E_{n}-E_{1}\right)},
$$

where $V_{n, 1}$ is a matrix element of the virial operator given by (32).

Let us discuss (36) and (37). We stress that they directly demonstrate that there is a finite probability,

$$
P_{n}=\left|a_{n}\right|^{2}=\left[\frac{\phi(R)}{c^{2}}\right]^{2}\left(\frac{V_{n, 1}}{E_{n}-E_{1}}\right)^{2}, \quad n \neq 1,
$$

that, at $t>0$, an electron occupies $n$th $(n \neq 1)$ energy level, which breaks the expected Einstein's equation, $m_{p}^{g}=m_{e}+$ $E_{1} / c^{2}$. In fact, this means that quantum measurement of passive gravitational mass (i.e., weight in the gravitational field (2)) in a quantum state with a definite energy (27) gives the quantized values (see (34)), corresponding to the probabilities (33) and (38), which are equal. (Note that, as it follows from quantum mechanics, we have to calculate wave function (35) in a linear approximation with respect to small parameter $\phi / c^{2}$ to obtain probabilities (38), which 
are proportional to $\left(\phi / c^{2}\right)^{2}$. A simple analysis shows that inclusion in (35) terms of the order of $\left(\phi / c^{2}\right)^{2}$ would change electron passive gravitational mass of the order of $\left(\phi / c^{2}\right) m_{e} \sim$ $10^{-9} m_{e}$, which is much smaller than the typical distance between the quantized values in (34), $\delta m_{p}^{g} \sim \alpha^{2} m_{e} \sim$ $10^{-4} m_{e}$.) We pay attention to the fact that small values of probabilities (33), (38), $P_{n} \sim 10^{-18}$, do not contradict the existing Eötvos type measurements [13], which have confirmed the equivalence principle with the accuracy of the order of $\delta \mathrm{m} / \mathrm{m} \sim 10^{-12}-10^{-13}$. As we mentioned in the previous section, for our case, it is crucial that the excited levels of a hydrogen atom spontaneously decay with time; therefore, one can detect the quantization law (34) by measuring electromagnetic radiation, emitted by a macroscopic ensemble of hydrogen atoms. The abovementioned optical method is much more sensitive than the Eötvos type measurements and we, therefore, believe that it will allow us to detect the breakdown of the equivalence between passive gravitational mass and energy, revealed in the paper.

\section{Suggested Realistic Experiment}

6.1. Hamiltonian. Let us consider a realistic experiment, which can be done on the Earth's orbit to detect photons, emitted by a macroscopic ensemble of hydrogen atoms with the following frequencies:

$$
\omega_{n, 1}=\frac{\left(E_{n}-E_{1}\right)}{\hbar} .
$$

As discussed above, these photons are the consequences of the quantization rule (34), breaking Einstein's equation for energy and passive gravitational mass. In the experiment we have to use a macroscopic ensemble of hydrogen atoms to make the number of the emitted photons to be large. More specifically, a tank of a pressurized hydrogen is located in small spacecraft or satellite and moved from a distant place, where gravitational potential is small, $|\phi(R)| \ll\left|\phi\left(R_{0}\right)\right|$, with constant velocity, $u \ll \alpha c$, towards the Earth. Note that the latter inequality allows us to disregard additional velocity-dependent corrections to the Hamiltonian (7) and (8), which can be derived from the Lagrangian of [3]. It is also important that each hydrogen atom is at rest with respect to the spacecraft (satellite), which means that gravitational force is compensated by some forces of nongravitational nature. Note that this changes a little the allowed frequencies (39). Nevertheless, the latter effect is out of our current consideration, since it is possible to show [14] that the changes of the frequencies (39) are less than the existing accuracies of their measurements. Other words, each hydrogen atom does not feel gravitational acceleration, $\mathbf{g}$, but rather feels timedependent gravitational potential, $\phi(R-u t)$. Therefore, each hydrogen atom is affected by the following time-dependent Hamiltonian:

$$
\widehat{H}=\frac{\widehat{\mathbf{p}}^{2}}{2 m_{e}}-\frac{e^{2}}{r}+\frac{\phi(R-u t)-\phi(R)}{c^{2}}\left[m_{e}+3 \frac{\widehat{\mathbf{p}}^{2}}{2 m_{e}}-2 \frac{e^{2}}{r}\right] .
$$

(For more rigorous derivation of (40), see [6].)
6.2. Photon Emission and Mass Quantization. Here, we describe the suggested realistic experiment in more detail. We consider a hydrogen atom to be in its ground state, at $t=0$, and located at distance $R$ from a center of the Earth, where the gravitational potential is small. The wave function of a ground state, corresponding to the Hamiltonian (7) and (8), can be written as

$$
\begin{aligned}
\widetilde{\Psi}_{1}(r, t)= & \left(1-\frac{\phi}{c^{2}}\right)^{3 / 2} \Psi_{1}\left[\left(1-\frac{\phi}{c^{2}}\right) r\right] \\
& \times \exp \left[\frac{-i m_{e} c^{2}\left(1+\phi / c^{2}\right) t}{\hbar}\right] \\
& \times \exp \left[\frac{-i E_{1}\left(1+\phi / c^{2}\right) t}{\hbar}\right],
\end{aligned}
$$

where $\phi=\phi(R)$. At arbitrary moment of time, $t>0$, electron wave function and time-dependent perturbation for the Hamiltonian (7) and (8) in inertial coordinate system, related to the spacecraft (satellite), can be expressed as

$$
\begin{aligned}
\widetilde{\Psi}(r, t)= & \left(1-\frac{\phi}{c^{2}}\right)^{3 / 2} \sum_{n=1}^{\infty} \widetilde{a}_{n}(t) \Psi_{n}\left[\left(1-\frac{\phi}{c^{2}}\right) r\right] \\
& \times \exp \left[\frac{-i m_{e} c^{2}\left(1+\phi / c^{2}\right) t}{\hbar}\right] \\
& \times \exp \left[\frac{-i E_{n}\left(1+\phi / c^{2}\right) t}{\hbar}\right], \\
\widehat{U}(\mathbf{r}, R, t) & =\frac{\phi(R-u t)-\phi(R)}{c^{2}}\left(3 \frac{\widehat{\mathbf{p}}^{2}}{2 m_{e}}-2 \frac{e^{2}}{r}\right) .
\end{aligned}
$$

Application of the time-dependent quantum mechanical perturbation theory [9] gives the following solutions for functions $\widetilde{a}_{n}(t)$ in $(42)$ :

$$
\begin{aligned}
& \tilde{a}_{n}(t)=-\frac{V_{n, 1}}{\hbar \omega_{n, 1} c^{2}} \\
& \times\left\{[\phi(R-u t)-\phi(R)] \exp \left(i \omega_{n, 1} t\right)\right. \\
&\left.\quad+\frac{u}{i \omega_{n, 1}} \int_{0}^{t} \frac{d \phi(R+u t)}{d R} d\left[\exp \left(i \omega_{n, 1} t\right)\right]\right\}, \\
& n \neq 1,
\end{aligned}
$$

where $V_{n, 1}$ and $\omega_{n, 1}$ are given by (32) and (39). It is important that under the suggested experiment the following inequality is obviously fulfilled:

$$
u \ll \omega_{n, 1} R \sim \alpha c\left(\frac{R_{0}}{r_{B}}\right) \sim 10^{13} c
$$


therefore, we can disregard the second term in the amplitude (44):

$$
\tilde{a}_{n}(t)=-\frac{V_{n, 1}}{\hbar \omega_{n, 1} c^{2}}[\phi(R-u t)-\phi(R)] \exp \left(i \omega_{n, 1} t\right),
$$

$n \neq 1$.

Since $|\phi(R)| \ll|\phi(R-u t)|$, we can write probabilities, corresponding to amplitudes of (46), in the following way:

$$
\widetilde{P}_{n}(t)=\left(\frac{V_{n, 1}}{\hbar \omega_{n, 1}}\right)^{2} \frac{\phi(R-u t)^{2}}{c^{4}}=\left(\frac{V_{n, 1}}{E_{n}-E_{1}}\right)^{2}\left[\frac{\phi\left(R^{\prime}\right)}{c^{2}}\right]^{2},
$$

where $R^{\prime}=R-u t$. It is important that the probabilities (47) depend only on gravitational potential, $\phi^{\prime}=\phi\left(R^{\prime}\right)$, in the final position of a spacecraft (satellite). Moreover, they coincide with the probabilities, obtained in both thought experiments (see (33) and (38)). This allows us to clarify their physical meaning. Indeed, since the probabilities (47), (33), and (38) are equal, we can conclude that all photons, emitted by a macroscopic ensemble of hydrogen atoms during the suggested realistic experiment, correspond to the breakdown of Einstein's equation for passive gravitation mass due to quantization of the mass (34). As we discussed above, the excited levels spontaneously decay with time and, therefore, it is possible to observe the quantization law (34) indirectly by measuring electromagnetic radiation from a macroscopic ensemble of the atoms. In this case, (47) gives probabilities that a hydrogen atom emits a photon with frequencies (39) during the time interval $t$. (We note that dipole matrix elements for $n S \rightarrow 1 S$ quantum transitions are zero. Nevertheless, the corresponding photons can be emitted due to quadrupole effects.)

Let us estimate the probabilities (47):

$$
\widetilde{P}_{n}=\left(\frac{V_{n, 1}}{E_{n}-E_{1}}\right)^{2} \frac{\phi^{2}\left(R^{\prime}\right)}{c^{4}} \simeq 0.49 \times 10^{-18}\left(\frac{V_{n, 1}}{E_{n}-E_{1}}\right)^{2},
$$

where, in (48), we use the following numerical values of the Earth's mass, $M \simeq 6 \times 10^{24} \mathrm{~kg}$, and its radius, $R_{0} \simeq 6.36 \times$ $10^{6} \mathrm{~m}$. It is important that although the probabilities (47) and (48) are small, the number of photons, $N$, emitted by macroscopic ensemble of the atoms, can be large since the factor $V_{n, 1}^{2} /\left(E_{n}-E_{1}\right)^{2}$ is of the order of unity. For instance, for 1000 moles of hydrogen atoms, $N$ is estimated as

$$
\begin{gathered}
N_{n, 1}=2.95 \times 10^{8}\left(\frac{V_{n, 1}}{E_{n}-E_{1}}\right)^{2}, \\
N_{2,1}=0.9 \times 10^{8},
\end{gathered}
$$

which can be experimentally detected, where $N_{n, 1}$ stands for a number of photons, emitted with frequency $\omega_{n, 1}=\left(E_{n}-E_{1}\right) / \hbar$ (see (39)).

\section{Active Gravitational Mass in Classical Physics}

Here, we introduce active gravitational mass for a classical model of a hydrogen atom. Suppose that we have a heavy positively charged particle (i.e., proton) with bare mass $m_{p}$ and light negatively charged particle (i.e., electron) with bare mass $m_{e}$, where $m_{p} \gg m_{e}$. At large distances, $R \gg r_{B}$, from the atom, gravitational potential in the first approximation is

$$
\phi(R)=-G \frac{m_{p}+m_{e}}{R},
$$

where we do not take into account kinetic and Coulomb potential energies contributions. Since $m_{p} \gg m_{e}$, we below disregard kinetic energy of proton and consider it as a center of mass of the atom. The next step is to define how kinetic and Coulomb potential energies of electron contribute to the electron active gravitational mass. To be more specific, we define active gravitational mass of the atom from gravitational potential acting on a small test body at rest at distances much high than the "size" of the atom, $r_{B}$. For simplicity, we prescribe potential and kinetic energies to electron and, therefore, consider corrections to electron gravitational mass. It is possible to show from general theory of a weak gravitational field $[1,13]$ that gravitational potential in our case can be written as

$$
\phi(R, t)=-G \frac{m_{p}+m_{e}}{R}-G \int \frac{\Delta T_{\alpha \alpha}^{k i n}(t, \mathbf{r})+\Delta T_{\alpha \alpha}^{p o t}(t, \mathbf{r})}{c^{2} R} d^{3} \mathbf{r},
$$

where $\Delta T_{\alpha \beta}^{k i n}(t, \mathbf{r})$ and $\Delta T_{\alpha \beta}^{p o t}(t, \mathbf{r})$ are changes of stress-energy tensor component $T_{\alpha \beta}(t, \mathbf{r})$ due to kinetic and Coulomb potential energies, respectively. (Note that in (51) and everywhere below we disregard the so-called retardation effects.) Therefore, in the second approximation in $1 / c^{2}$, active electron gravitational mass can be written as

$$
m_{a}^{g}=m_{e}+\frac{1}{c^{2}} \int\left[\Delta T_{\alpha \alpha}^{k i n}(t, \mathbf{r})+\Delta T_{\alpha \alpha}^{p o t}(t, \mathbf{r})\right] d^{3} \mathbf{r}
$$

Let us write the standard expression for stress-energy tensor of a moving point particle without electrical charge $[1,13]$ :

$$
T^{\alpha \beta}(\mathbf{r}, t)=\frac{m v^{\alpha}(t) v^{\beta}(t)}{\sqrt{1-v^{2} / c^{2}}} \delta^{3}\left[\mathbf{r}-\mathbf{r}_{\mathbf{p}}(t)\right],
$$

where $v^{\alpha}$ is a four-velocity, $\delta^{3}[\cdots]$ is the three-dimensional Dirac's delta function, and $\mathbf{r}_{\mathbf{p}}(t)$ is a trajectory of the particle in three-dimensional space. It is easy to show by means of (53) that at low enough velocity, $v \ll c$,

$$
\Delta T_{\alpha \alpha}^{k i n}=3 \frac{m v^{2}}{2} .
$$

The standard expression for stress-energy tensor of electromagnetic field [1] can be written as

$$
T_{e m}^{\mu \nu}=\frac{1}{4 \pi}\left[F^{\mu \alpha} F_{\alpha}^{\nu}-\frac{1}{4} \eta^{\mu \nu} F_{\alpha \beta} F^{\alpha \beta}\right],
$$


where $F^{\alpha \beta}$ is the so-called electromagnetic field tensor and $\eta_{\alpha \beta}$ is metric of the Minkowski spacetime. This expression can be significantly simplified in our case, where only electrical field is present. As a result, we obtain the following formula for change of the stress-energy tensor in the presence of the Coulomb potential energy:

$$
\Delta T_{\alpha \alpha}^{p o t}=-2 \frac{e^{2}}{r}
$$

Therefore, the total electron active gravitational mass can be written in the same way as the electron passive mass:

$$
\begin{aligned}
m_{a}^{g}= & m_{e}+\frac{\left(\left(m_{e} \mathbf{v}^{2} / 2\right)-\left(e^{2} / r\right)\right)}{c^{2}} \\
& +\frac{\left(\left(2\left(m_{e} \mathbf{v}^{2} / 2\right)\right)-\left(e^{2} / r\right)\right)}{c^{2}},
\end{aligned}
$$

where the last term is the virial one. Since the virial term changes with time, we come to the conclusion that active gravitational mass of a classical body changes with time. Nevertheless we can introduce averaged over time active gravitational mass, which occurs to be equivalent to energy $[3,4]$, since the averaged over time virial term is zero:

$$
\begin{aligned}
\left\langle m_{a}^{g}\right\rangle= & m_{e}+\frac{\left\langle\left(\left(\widehat{\mathbf{p}}^{2} / 2 m_{e}\right)\right)-\left(e^{2} / r\right)\right\rangle_{t}}{c^{2}} \\
& +\frac{\left\langle\left(2\left(m_{e} \mathbf{v}^{2} / 2\right)\right)-\left(e^{2} / r\right)\right\rangle_{t}}{c^{2}} \\
= & m_{e}+\frac{E}{c^{2}} .
\end{aligned}
$$

\section{Equivalence of the Expectation Values of Active Gravitational Mass and Energy for Stationary States}

Here, we use the so-called semiclassical approach to the general relativity (see, e.g., $[16,17])$, where Einstein's gravitational equation can be written as

$$
R_{\mu \nu}-\frac{1}{2} R g_{\mu \nu}=\frac{8 \pi G}{c^{2}}\left\langle\widehat{T}_{\mu \nu}\right\rangle
$$

where the last term represents the expectation value of quantum stress-energy operator of the matter. In our case, expression for active gravitational mass (57) can be represented as the following Hamiltonian:

$$
\begin{aligned}
m_{a}^{g}= & m_{e}+\frac{\left(\left(\mathbf{p}^{2} / 2 m_{e}\right)-\left(e^{2} / r\right)\right)}{c^{2}} \\
& +\frac{\left(\left(2\left(\mathbf{p}^{2} / 2 m_{e}\right)\right)-\left(e^{2} / r\right)\right)}{c^{2}},
\end{aligned}
$$

which can be easily quantized:

$$
\begin{aligned}
\widehat{m}_{a}^{g}= & m_{e}+\frac{\left(\left(\widehat{\mathbf{p}}^{2} / 2 m_{e}\right)-\left(e^{2} / r\right)\right)}{c^{2}} \\
& +\frac{\left(\left(2\left(\widehat{\mathbf{p}}^{2} / 2 m_{e}\right)\right)-\left(e^{2} / r\right)\right)}{c^{2}} .
\end{aligned}
$$

Therefore, in the framework of semiclassical theory of gravity, the expectation value of active gravitational mass, corresponding to macroscopic ensemble of the atoms with each of them being in its ground state, is equal to

$$
\begin{aligned}
\left\langle\widehat{m}_{a}^{g}\right\rangle= & m_{e}+\frac{\left\langle\left(\hat{\mathbf{p}}^{2} / 2 m_{e}\right)-\left(e^{2} / r\right)\right\rangle}{c^{2}} \\
& +\frac{\left\langle\left(2\left(\hat{\mathbf{p}}^{2} / 2 m_{e}\right)\right)-\left(e^{2} / r\right)\right\rangle}{c^{2}} \\
= & m_{e}+\frac{E_{1}}{c^{2}} .
\end{aligned}
$$

Thus, we conclude that the expectation values of active gravitational mass and energy are equivalent for stationary quantum states.

\section{Inequivalence between Active Gravitational Mass and Energy for Superpositions of Stationary States}

In the previous section we have established the equivalence for the expectation values of active gravitational mass and energy for stationary quantum states. Below, we study if such equivalence survives for superpositions of stationary quantum states. As in Section 4, we consider the simplest superposition of the ground and first excited s-wave states in a hydrogen atom, where electron wave function can be written as

$$
\Psi_{1,2}(r, t)=\frac{1}{\sqrt{2}}\left[\Psi_{1}(r) \exp \left(-i E_{1} t\right)+\Psi_{2}(r) \exp \left(-i E_{2} t\right)\right] .
$$

As we discussed this before, the expectation value of energy for the wave function (63) does not depend on time. Nevertheless, the expectation value of active gravitational mass operator (61) oscillates with time and has the following form:

$$
\left\langle\widehat{m}_{a}^{g}\right\rangle=m_{e}+\frac{E_{1}+E_{2}}{2 c^{2}}+\frac{V_{1,2}}{c^{2}} \cos \left[\frac{\left(E_{1}-E_{2}\right) t}{\hbar}\right],
$$

which coincides with (24), describing time-dependent oscillations of passive gravitational mass, where matrix element of the virial operator, $V_{1,2}$, is given by (25). As we discussed before such oscillations are of a pure quantum origin and do not have classical analogs. They directly demonstrate inequivalence of active gravitational mass and energy at a macroscopic level. Nevertheless, in the same way as in Section 4, we can introduce modified equivalence principle 
for the expectation values of active gravitational mass and energy by means of averaging of (64) over time:

$$
\left\langle\left\langle\widehat{m}_{a}^{g}\right\rangle\right\rangle_{t}=m_{e}+\frac{\left(E_{1}+E_{2}\right)}{2 c^{2}}=\left\langle\frac{E}{c^{2}}\right\rangle .
$$

As we stressed in Section 4, such averaging procedure is principally different from that in (1) and (58) and does not have classical analogs.

\section{Conflict of Interests}

The authors declare that there is no conflict of interests regarding the publication of this paper.

\section{Acknowledgments}

The authors are thankful to N. N. Bagmet (Lebed), V. A. Belinski, Steven Carlip, Douglas Singleton, and V. E. Zakharov for useful discussions. This work was supported by the NSF under Grant DMR-1104512.

\section{References}

[1] L. D. Landau and E. M. Lifshitz, The Classical Theory of Fields, Butterworth-Heineman, Amsterdam, The Netherlands, 4th edition, 2003.

[2] C. W. Misner and P. Putnam, "Active gravitational mass," Physical Review, vol. 116, no. 4, pp. 1045-1046, 1959.

[3] K. Nordtvedt, "Post-Newtonian gravity: its theory-experiment interface," Classical and Quantum Gravity, vol. 11, no. 6, article A119, 1994.

[4] S. Carlip, "Kinetic energy and the equivalence principle," American Journal of Physics, vol. 66, no. 5, pp. 409-413, 1998.

[5] A. G. Lebed, "Breakdown of the equivalence between passive gravitational mass and energy for a quantum body," in Proceedings of the Marcel Grossmann Meeting-13, Sweden, Stockholm, July 2012.

[6] A. G. Lebed, "Is gravitational mass of a composite quantum body equivalent to its energy?" Central European Journal of Physics, vol. 11, no. 8, pp. 969-976, 2013.

[7] A. G. Lebed, "Breakdown of the equivalence between gravitational mass and energy for a composite quantum body," Journal of Physics: Conference Series. In press.

[8] F. Schwabl, Advanced Quantum Mechanics, Springer, Berlin, Germany, 3rd edition, 2005.

[9] D. Park, Introduction to the Quantum Theory, Dover, New York, NY, USA, 3rd edition, 2005.

[10] W. Lucha and F. F. Schoberl, "Relativistic virial theorem," Physical Review Letters, vol. 64, no. 23, pp. 2733-2735, 1990.

[11] R. Colella, A. W. Overhauser, and S. A. Werner, "Observation of gravitationally induced quantum interference," Physical Review Letters, vol. 34, no. 23, pp. 1472-1474, 1975.

[12] V. V. Nesvizhevsky, H. G. Börner, A. K. Petukhov et al., "Quantum states of neutrons in the Earth's gravitational field," Nature, vol. 415, no. 6869, pp. 297-299, 2002.

[13] C. W. Misner, K. S. Thorne, and J. A. Wheeler, Gravitation, W.H. Freeman and Co., New York, NY, USA, 1973.

[14] A. G. Lebed, in a preparation.
[15] E. Fischbach, B. S. Freeman, and W.-K. Cheng, "General-relativistic effects in hydrogenic systems," Physical Review D, vol. 23, no. 10, pp. 2157-2180, 1981.

[16] N. D. Birrell and P. C. W. Davis, Quantum Fields in Curved Space, Cambridge University Press, Cambridge, UK, 1982.

[17] D. N. Page and C. D. Geilker, "Indirect evidence for quantum gravity," Physical Review Letters, vol. 47, no. 14, pp. 979-982, 1981. 

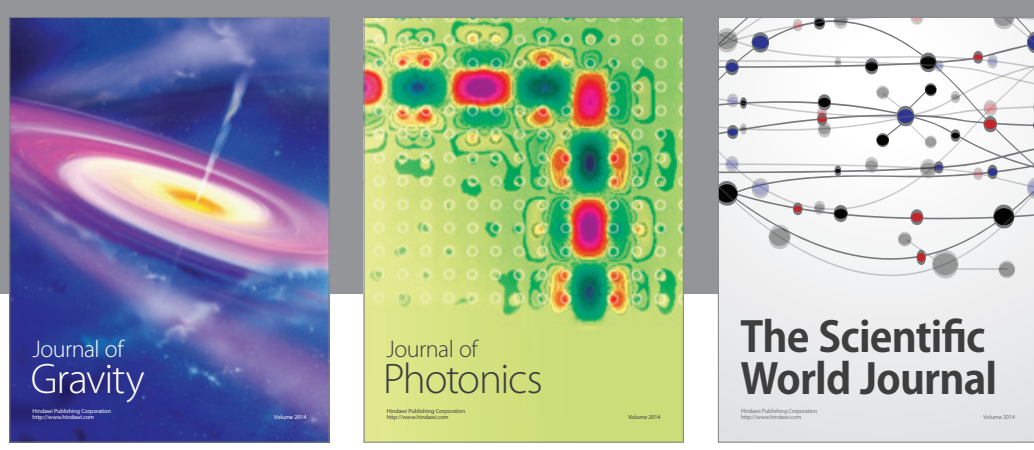

The Scientific World Journal
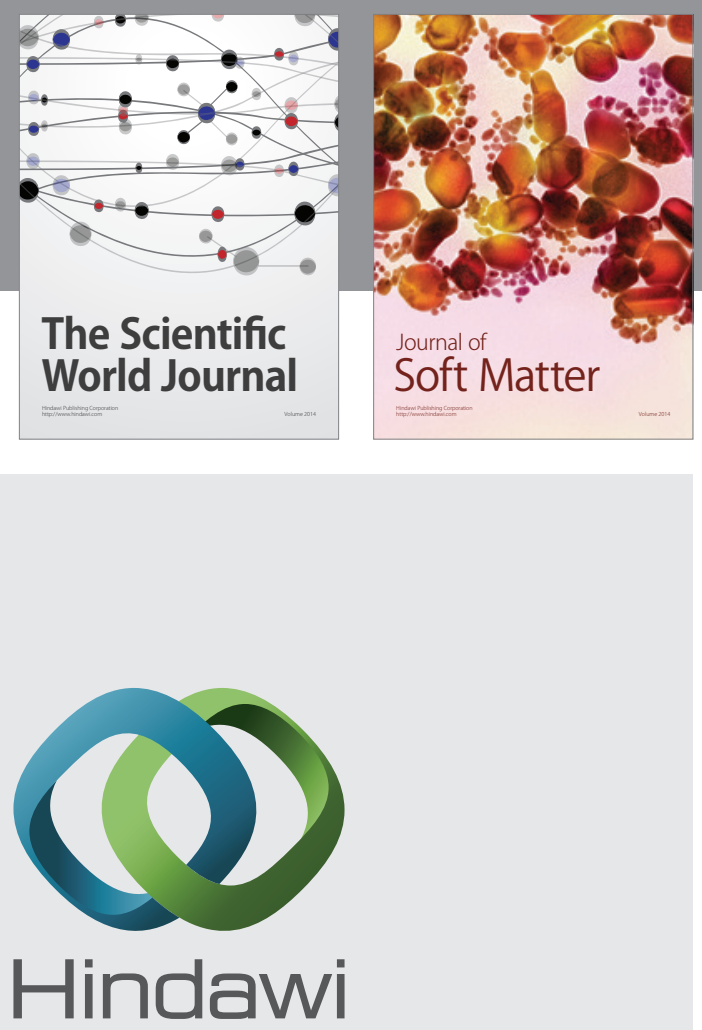

Submit your manuscripts at

http://www.hindawi.com

nternational Journal of

Statistical Mechanics
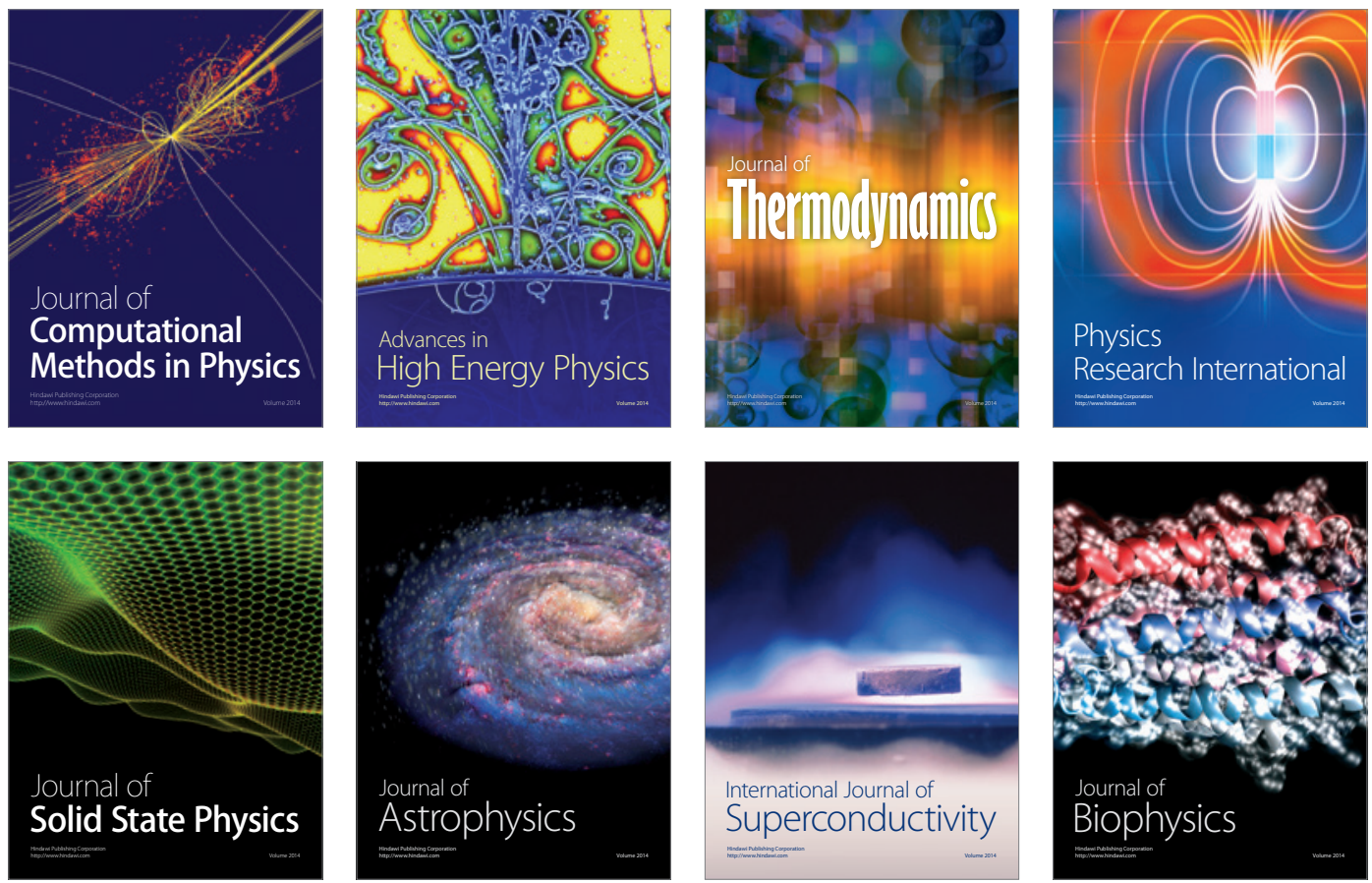
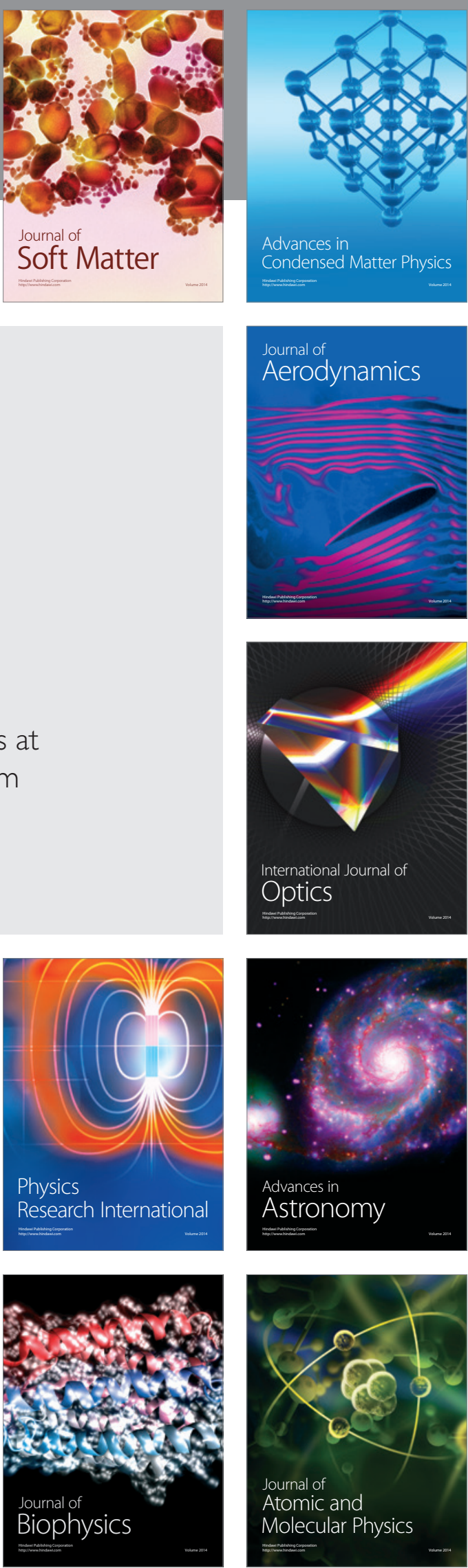